\title{
Analysis of CRP, Antithrombin, Fibrinogen, and Hematological Changes in 433 Patients with PTE
}

\author{
Yu-Yan Wu* \\ Yong Tan* \\ Min Yan \\ Zhen Cheng \\ Xiang-Tao Pan
}

Department of Hematology, Taicang Hospital of Soochow University, Taicang, Jiangsu, People's Republic of China

*These authors contributed equally to this work
Correspondence: Xiang-Tao Pan Department of Hematology, Taicang Hospital of Soochow University, No. 58. of South Changsheng Road, Taicang, jiangsu, People's Republic of China Tel +86 I89I5768003

Email Panxt99xt@email.cn
Objective: To investigate the characteristics of coagulation indicators, such as C-reactive protein (CRP), antithrombin (AT), and fibrinogen (FB) in patients with pulmonary thromboembolism (PTE) and their relationship with PTE.

Methods: The clinical characteristics, CRP, AT, FB, plasma D-dimer (D-D) and platelet (PLT) counts, and blood coagulation indicators of 433 patients with PTE diagnosed by computed tomographic pulmonary angiography (CTPA) were comprehensively analyzed, and the relevant clinical significance was discussed.

Results: The total incidence of PTE was 1.60\% (433/270,983 cases), and the age group with the highest incidence was over 60 years old. D-D increased in $412(95.2 \%)$ patients. PLT was normal in 331 (76.4\%) cases, but decreased in 53 (12.3\%) cases and increased in $49(11.3 \%)$ cases. CRP increased in 76.0\% (288/379 cases) and was normal in 24.0\% (91/379 cases). AT decreased in $109(25.2 \%)$ cases and FB increased in $102(23.6 \%)$ cases. There were no significant changes in other coagulation indexes. CRP was positively correlated with PLT and $\mathrm{FB}(\mathrm{r}=0.1026, \mathrm{t}=2.0118, \mathrm{P}<0.05$, and $\mathrm{r}=0.5360, \mathrm{t}=12.2963, \mathrm{P}<0.01$, respectively). CRP was negatively correlated with AT $(r=-0.2005, \mathrm{t}=4.0100, \mathrm{P}<0.01)$.

Conclusion: The incidence of PTE was higher in hospitalized patients. Most patients with PTE have increased CRP and FB and decreased AT, and these levels may be related to the occurrence of PTE.

Keywords: pulmonary thromboembolism, C-reactive protein, antithrombin, fibrinogen, D-dimer, platelets

\section{Introduction}

Venous thromboembolism (VTE), including pulmonary thromboembolism (PTE) and deep venous thrombosis (DVT), has a serious impact on patients' quality of life and can even cause disability and life-threatening complications. ${ }^{1}$ In recent years, there has been an increasing trend of VTE. PTE and DVT are different manifestations of the disease process in other parts of the body at various stages of the disease. The majority of PTE thrombosis comes from DVT. In clinical practice, PTE is acute and severe with high mortality. ${ }^{2-4}$ Therefore, it is of great significance to study the pathogenesis, changes in clinical and laboratory indicators, which can be easily determined by simple and easy measurement, and effective prevention and treatment measures of PTE. ${ }^{1,5}$ The importance of several clinical and laboratory variables has been proved in PTE patients. ${ }^{6-8}$ In addition, elevated levels of biochemical markers such as troponin, brain natriuretic peptide (BNP), NTproBNP, H-FABP (heart type fatty acid binding protein), myoglobin, and white blood cell (WBC) count have been shown to predict adverse events. It is vital to 
decrease patient mortality and morbidity rates by immediate initiation of anticoagulation and/or thrombolytic therapy, especially in relatively stable patients, ${ }^{9}$ so new risk markers are being investigated. In this paper, the clinical coagulation indexes and hematological characteristics of 433 cases of PTE were comprehensively analyzed, and the relationship between the occurrence and development of PTE was discussed. This study aimed to evaluate the prognostic role of hematological changes in PTE.

\section{Materials and Methods}

\section{Patients' Characteristics}

From January 1, 2015, to December 31, 2019, a total of 270,983 patients were discharged from our hospital, including 433 patients with PTE (excluding repeated hospitalization of the same patient). There were 259 males and 174 females, aged 18.0-96.0 years old, with an average age of 71.3 years. A total of 28 healthy subjects were selected as the control group, with 18 males and 10 females, aged 28-43 years with an average age of 38.5 years. This study was conducted with approval from the Ethics Committee of Taicang Hospital of Soochow University (No. 2021-KY-156), the signed informed consent requirement was waved. This study was conducted in accordance with the declaration of Helsinki.

\section{Study Methods}

The VTE network of our hospital provided all relevant medical records collected through the medical record center for the data of hospitalized VTE patients discharged from our hospital from January 1, 2015, to December 31, 2019. We reviewed ultrasounds or computed tomographic pulmonary angiography (CTPA), plasma D-dimer (D-D), C-reactive protein (CRP), antithrombin III (AT-III, referred to as AT), fibrinogen (FB), hemoglobin $(\mathrm{Hb})$, and white blood cell (WBC) and platelet (PLT) counts. Finally, these 433 cases of PTE were included in this study.

The following information was recorded: medical record number, name, gender, age, inpatient department, primary disease, inducing factors, occurrence location, preventive measures, related test results, treatments, and prognosis.

\section{Statistical Methods}

Normally distributed variables were expressed as mean \pm SD and were compared using a Student's $t$-test. Categorical variables were expressed in percentages and compared using the $\chi^{2}$ test. All P-values were two-tailed, and values less than 0.05 were considered statistically significant. Analyses were carried out using SPSS software (version 23.0).

\section{Results}

\section{Incidence and Clinical Features}

From 2015 to 2019, the total number of discharged cases was 270,983 , with $433(1.60 \%)$ cases of PTE, including 350 cases of simple PTE $(80.80 \%)$ and 83 cases of PTE combined with DVT (19.2\%). There were 15 patients (3.5\%) younger than 40 years old and $362(83.6 \%)$ older than 60. According to each department, at the time of the first occurrence of PTE, the non-surgical departments $(360 / 145,491,0.25 \%)$ had significantly more cases than the surgical departments $(73 / 125,492,0.06 \%) \quad\left(\chi^{2}=\right.$ 151.29, $\mathrm{P}<0.001)$.

In 122 cases, PTE occurred in the bilateral pulmonary artery, the right pulmonary artery in 184 cases, and the left pulmonary artery trunk in 127 cases. The primary reasons for hospitalization were malignant tumors in 106 cases, 87 had traumatic fractures, 69 had operations, and 101 were infection-related. Second, most of them had coronary heart disease, cerebral infarction, or kidney disease, and a few had immune thrombocytopenia, systemic lupus erythematosus, or pesticide poisoning. There were 183 smokers, 251 patients with hypertension, 198 with diabetes mellitus, and 130 had hypertension combined with diabetes. The main predisposing factors are immobilization or bed rest due to disease, hypercoagulant state and catheterization, etc. Most patients have multiple predisposing or high-risk factors at the same time. The prognosis of patients with PTE is generally good as long as they receive timely diagnosis and treatment. In this group, $352(81.29 \%)$ patients were cured or improved, $73(16.86 \%)$ deteriorated or were offer to leave the hospital, and $8(1.85 \%)$ died (Table 1$)$.

\section{Results of Plasma D-D}

At the time of onset, D-D was $230.00-80,000.00 \mu \mathrm{g} / \mathrm{L}$. It was increased in 412 cases, normal in 20, and there was no record in one case. Among the 20 normal cases, the level of D-D in 13 cases were higher at the onset of PTE.

\section{Results of Plasma CRP}

The CRP was recorded in 379 cases. Fifty-four cases had no CRP records. The levels of CRP ranged from $0.3-813.2 \mathrm{mg} / \mathrm{L}$. Two hundred eighty-eight cases had elevated CRP $(>10.0 \mathrm{mg} / \mathrm{L})$, and $91 \mathrm{had}$ normal CRP $(0.1-10.0 \mathrm{mg} / \mathrm{L})$. 
Table I The Characteristics of 433 PTE Patients

\begin{tabular}{|c|c|c|}
\hline Items & Classification & Cases (n) \\
\hline \multirow[t]{2}{*}{ Gender } & Male & 259 \\
\hline & Female & 174 \\
\hline \multirow[t]{3}{*}{ Age } & $<40$ & 15 \\
\hline & $46-60$ & 56 \\
\hline & $>60$ & 362 \\
\hline \multirow[t]{3}{*}{ Pulmonary artery } & Bilateral & 122 \\
\hline & Right & 184 \\
\hline & Left & 127 \\
\hline \multirow[t]{2}{*}{ Smoking } & Yes & 183 \\
\hline & No & 250 \\
\hline \multirow[t]{2}{*}{ Hypertension } & Yes & 251 \\
\hline & No & 182 \\
\hline \multirow[t]{2}{*}{ Diabetes } & Yes & 198 \\
\hline & No & 235 \\
\hline Hypertension \& & Yes & 130 \\
\hline Diabetes & No & 303 \\
\hline \multirow[t]{3}{*}{ Prognosis } & Healed & 352 \\
\hline & Unhealed & 73 \\
\hline & Death & 8 \\
\hline
\end{tabular}

\section{Results of Plasma AT, FB, and Other Coagulation Indicators}

The results of AT, FB, prothrombin time (PT), activated partial thromboplastin time (APTT), thrombin time (TT), and international normalized ratio (INR) are shown in Table 2.

\section{Results of Hematological Indicators}

The indicators of $\mathrm{Hb}$, WBC, and PLT are shown in Table 3. Hb was normal in 275 patients, decreased in 152 , and 6 had increased levels. PLT was normal in 328 patients, 53 had decreased levels, and it increased in 52 . WBC was normal in 293 patients, decreased in 35, and increased in 105 .

\section{Correlation Analysis}

Correlation Analysis Between CRP and PLT, D-D, AT, and FB

CRP was positively correlated with PLT and FB ( $\mathrm{r}=$ $0.1026, \mathrm{t}=2.0118, \mathrm{P}<0.05$, and $\mathrm{r}=0.5360, \mathrm{t}=$ $12.2936, \mathrm{P}<0.001$, respectively), and negatively correlated with AT $(\mathrm{r}=-0.2005, \mathrm{t}=4.0100, \mathrm{P}<0.01)$. There was no correlation between CRP and D-D $(r=0.0791, t=$ $1.5510, \mathrm{P}>0.05)$.

Correlation Analysis Between AT, FB, PLT, and D-D FB was positively correlated with PLT $(r=0.2110, t=$ 4.2200, $\mathrm{P}<0.01)$ but had no correlation with $\mathrm{D}-\mathrm{D}(\mathrm{r}=-$ $0.0658, \mathrm{P}>0.05)$. AT had no correlation with PLT and $\mathrm{D}-\mathrm{D}(\mathrm{r}=0.0798$ and $\mathrm{r}=0.0066$, respectively, $\mathrm{P}>0.05$ for all).

\section{Discussion}

Vascular endothelial injury, slow blood flow rate, and a hypercoagulable state are the three main factors of thrombosis, which are more likely to occur in patients with diabetes, hypertension, malignant tumors, and other primary diseases. ${ }^{1}$ Surgery and targeted therapy can also increase the risk of VTE. ${ }^{1,2}$ The annual incidence of PTE in the United States is $1.17 \%$, and the incidence reported in China of hospitalized patients is $1.45 \% .^{3}$ In this study, the incidence is $1.60 \%$, which is the same as in previous studies. In this research, the common sites of embolism, clinical manifestations, and prognosis of patients with PTE were consistent with those reported in the literature. ${ }^{1-4}$ It has been reported that the mortality rate of untreated patients is as high as $25.0-30.0 \%$. ${ }^{4,5}$ Therefore, attention should be paid to PTE in clinical practice. D-D was increased in $95.2 \%$ of the patients. Although D-D was lower than normal at the occurrence of PTE in a few patients, the level was significantly higher than at admission, indicating that dynamic observation of the changes in D-D was of particular significance for the diagnosis of PTE. ${ }^{10}$ In addition, there was no correlation between

Table 2 Detection Results of Coagulation Indexes in 433 PTE Patients

\begin{tabular}{|l|l|l|l|l|l|l|}
\hline Items & PT (Sec) & APTT (Sec) & TT (Sec) & AT (Sec) & FB (Sec) & INR (Sec) \\
\hline Range & $10.0-91.6$ & $16.3-78.9$ & $13.6-26.0$ & $33.0-156.0$ & $0.4-6.0$ & $0.80-4.00$ \\
Mean value & $13.8 \pm 9.6$ & $30.8 \pm 9.7$ & $17.8 \pm 5.1$ & $85.9 \pm 28.6$ & $3.47 \pm 1.28$ & $1.16 \pm 0.55$ \\
Decrease(n) & 0 & 0 & 2 & 109 & 3 & 1 \\
Normal(n) & 396 & 397 & 413 & 318 & 328 & 412 \\
Rise(n) & 37 & 36 & 18 & 6 & 102 & 20 \\
Normal reference value & $9.6-14.3$ & $23.3-32.5$ & $14.0-21.0$ & $75.0-130.0$ & $1.7-4.1$ & $0.85-1.25$ \\
\hline
\end{tabular}


Table 3 The Results of Hematological Indexes in 433 PTE Patients and Control Group

\begin{tabular}{|l|l|l|l|}
\hline Items & PTE $(\mathbf{n}=\mathbf{4 3 3})$ & Control Group $(\mathbf{n}=\mathbf{2 8})$ & t \\
\hline $\mathrm{Hb}(\mathrm{g} / \mathrm{L})$ & $93.32 \pm 56.5 \mathrm{I}$ & $138.20 \pm 9.66$ & $3.2362 * *$ \\
$\mathrm{RDW}-\mathrm{CV}(\%)$ & $10.73 \pm 6.39$ & $12.66 \pm 0.37$ & $1.207 \mathrm{I}$ \\
$\mathrm{WBC}\left(\times 10^{9} / \mathrm{L}\right)$ & $6.63 \pm 6.03$ & $5.68 \pm 1.53$ & 0.6089 \\
$\mathrm{~N}\left(\times 10^{9} / \mathrm{L}\right)$ & $5.11 \pm 4.55$ & $3.24 \pm 1.18$ & 1.4489 \\
$\mathrm{~L}\left(\times 10^{9} / \mathrm{L}\right)$ & $0.88 \pm 0.83$ & $1.94 \pm 0.46$ & $5.0237 * *$ \\
$\mathrm{M}\left(\times 10^{9} / \mathrm{L}\right)$ & $0.44 \pm 0.43$ & $0.29 \pm 0.28$ & 1.3286 \\
$\mathrm{PLT}\left(\times 10^{9} / \mathrm{L}\right)$ & $193.26 \pm 90.23$ & $237.63 \pm 38.61$ & 1.1233 \\
$\mathrm{MPV}(\mathrm{fl})$ & $8.28 \pm 4.68$ & $10.48 \pm 0.90$ & 1.8553 \\
$\mathrm{PSD}(\%)$ & $9.93 \pm 5.91$ & $12.44 \pm 1.93$ & 1.6929 \\
$\mathrm{P}-\mathrm{LCR}(\%)$ & $24.13 \pm 15.18$ & $28.46 \pm 7.69$ & 1.1344 \\
\hline
\end{tabular}

Notes: ${ }^{* *} \mathrm{p}<0.01$, the rest are $\mathrm{p}>0.05$.

D-D and CRP, AT, and FB, indicating that D-D had little relationship with inflammatory and coagulation factors, indicating it was not the cause of PTE but a manifestation after the occurrence of PTE.

In the study, as many as $76.0 \%$ of patients had elevated $\mathrm{CRP}$, suggesting that it may be related to the occurrence of PTE. Some studies believe that CRP is related to arterial thrombosis, while its relationship with VTE is not clear. ${ }^{11}$ But more evidence shows that CRP is associated with PTE. In this study, AT decreased in $25.2 \%$ of patients and $\mathrm{FB}$ increased in $23.6 \%$, while PT, APTT, TT, and INR had no significant changes, which suggested that AT and FB were related to the occurrence of PTE. Further analysis showed that AT was negatively correlated with CRP, while FB was positively correlated, indicating that an increase of CRP led to a decrease of AT and an increase of $\mathrm{FB}$, which further supported that AT and FB were among the causes of PTE. It has been reported that inflammationstimulating factor CRP and tumor necrosis factor $\alpha$ $(\mathrm{TNF} \alpha)$ in immune diseases, like autoantibodies IgM, can promote blood coagulation, leading to elevated levels of a blood coagulation factor and the formation of $\mathrm{DVT}^{12}$ Asakuraet al also showed that CRP, TNF $\alpha$, and IL-6 stimulated an increase of coagulation factors, such as FB, in inflammatory diseases, resulting in the formation of DVT. ${ }^{13}$ Elevated CRP levels promote the transformation of FB into fibrin, which leads to an increase in fibrin levels, blood viscosity, and DVT. ${ }^{14}$ Esmon et al confirmed that elevated IL- 6 and CRP resulted in increased PLT, FB, and decreased AT. ${ }^{15}$ Zhang et al studied 182 patients with type 2 diabetes mellitus and found that IL-6, CRP, and FB with microvascular complications were higher than those without thrombus. CRP was positively correlated with FB. ${ }^{16}$ The results of the above literature are consistent with this study. It can be seen that decreased AT and increased FB caused by increased CRP are important factors leading to PTE occurrence.

As for the PLT count, $12.2 \%$ of patients had reduced levels, and $12.0 \%$ had increased levels, with varying variations, which may be related to the different inflammatory states of the patients. PLT is generally considered to be reduced in severe infections and elevated in chronic infections, such as tuberculosis, osteomyelitis, or subacute bacterial endocarditis (SBE). Previous studies showed that PLT decreased in $2 / 3$ of the patients with bacterial sepsis and significantly decreased in $1 / 3$ of the patients $(<30.0 \times$ $\left.10^{9 /} \mathrm{L}\right){ }^{17}$ This data shows that PLT has a low correlation with CRP, and previous studies ${ }^{15}$ also indicate that CRP can promote the increase of PLT. In addition, PLT is also positively correlated with $\mathrm{FB}$, which suggests that changes in PLT are related to inflammation, and an increase of the PLT count is associated with the occurrence of PTE. Of course, patients with reduced PLT count may also develop PTE, and it has been proven that reduced PLT cannot prevent the occurrence of PTE. ${ }^{15}$ In the study, five patients with immune thrombocytopenia had PLT counts below $80.0 \times 10^{9} / \mathrm{L}$, and the lowest one was $28.0 \times 10^{9} / \mathrm{L}$, indicating that PTE could also occur in patients with decreased PLT.

The levels of $\mathrm{Hb}$ and $\mathrm{WBC}$ vary. In this study, the $\mathrm{Hb}$ of patients with PTE was significantly lower than the control group $(\mathrm{P}<0.01)$, which was related to the increase of CRP. Our previous studies have confirmed that elevated IL-6 and CRP lead to increased expression of Hepcidin and lead to poor utilization of iron, and finally, anemia. ${ }^{18,19}$ Although the WBC, neutrophil, and monocyte counts were higher than in the control group, there was no statistical significance. According to this study and 
previous studies, the changes of $\mathrm{Hb}$ and $\mathrm{WBC}$ are not the causes of PTE. The actual relationship between the changes of lymphocytes and PTE still needs to be studied further.

In conclusion, patients with PTE have specific abnormal characteristic changes in coagulation and hematological indicators. Elevated CRP was found to cause a series of changes, such as decreased AT, increased FB and PLT, and is closely related to PTE occurrence. It is worth further study in the future.

\section{Ethics Approval and Consent to Participate}

This study was conducted with approval from the Ethics Committee of Taicang Hospital of Soochow University (No. 2021-KY-156), written informed consent was obtained from all participants.This study was conducted in accordance with the declaration of Helsinki.

\section{Consent for Publication}

All participants signed a document of informed consent.

\section{Disclosure}

The authors declare that they have no competing interests.

\section{References}

1. Ahmed AB, Koster A, Lance M, et al. Eouropean guidelines on perioperative venous thromboembolism prophylaxis: cardiovascularand thoracic surgery. Eur $J$ Anaesthesiol. 2018;35(2):84-89. doi:10.1097/EJA.0000000000000708

2. Afshari A, Ageno W, Ahmed A, et al. European guidelines on perioperative venous thromboembolism prophylaxis: executivesummary. Eur J Anaesthesiol. 2018;35(2):77-83. doi:10.1097/EJA.00000 00000000729

3. Yun JL, Li XY. [Research status of pathoepidemiology of venous thromboembolism]. Zhong Hua Lao Nian Xin Nao Xue Guan Bing Za Zhi. 2017;19(7):322-324. Chinese.

4. Zhang W, Wang DY, Cui D, et al. [Autopsy analysis of 12 cases of type 2 diabetes mellitus complicated with pulmonary embolism]. Zhong Guo LIn Chuang Yi Sheng Za Zhi. 2015;6(6):42-44. Chinese.
5. Shimi M, Allouche M, Ben Ahmed H, et al. Sudden death duo to pulmonary rmbolism in north Tunisia:37 cases study. Tunis Med. 2014;92(10):610-614.

6. Konstantinides SV, Torbicki A, Agnelli G, et al. ESC Committee for Practice Guidelines (CPG). Guidelines on the diagnosis and management of acute pulmonary embolism: the Task Force for the Diagnosis and Management of Acute Pulmonary Embolism of the European Society of Cardiology (ESC). Eur Heart J. 2014;35:3033-3069.

7. Goldhaber SZ, Visani L, De Rosa M. Acute pulmonary embolism: clinical outcomes in the International Cooperative Pulmonary Embolism Registry (ICOPER). Lancet. 1999;353:1386-1389. doi:10.1016/S0140-6736(98)07534-5

8. Donze J, Le Gal G, Fine MJ, et al. Prospective validation of the pulmonary embolism severity index. A clinical prognostic model for pulmonary embolism. Thromb Haemost. 2008;100:943-948. doi:10.1160/TH08-05-0285

9. Karatas MB, Ipek G, Onuk T, et al. Assessment of prognostic value of neutrophil to lymphocyte ratio and platelet to lymphocyte ratio in patients with pulmonary embolism. Acta Cardiol Sin. 2016;32 (3):313-320.

10. Karsy M, Azab MA, Harper J, et al. Evaluation of a D-dimer protocol for detection of venous thromboembolism. World Neurosurg. 2020;133:e774-e783. doi:10.1016/j.wneu.2019.09.160

11. Giuseppe L, Emmanuel JF, Martina M, et al. C-reactive protein and venous thromboembolism: causalor casual association? Clin Chem Lab Med. 2010;48(12):1693-1701.

12. Tamaki H, Khasnis A. Venous thromboembolism in systemic autoimmune diseases: a narrative review with emphasis on primary systemic vasculitides. Vasc Med. 2015;20(4):369-376. doi:10.1177/ 1358863X15573838

13. Asakura H. [Thrombosis in myeloproliferative neoplasms]. Rinsho Ketsueki. 2018;59(8):1034-1041. Japanese.

14. Mukubo Y, Kawamata M. Perioperative hypercoagulability in patients with rheumatoid arthritis: sonoclot study. J Anesth. 2004;18 (1):62-64. doi:10.1007/s00540-003-0206-4

15. Esmon CT. Inflammation and thrombosis. J Thromb Haemost. 2003;1 (7):1343-1348. doi:10.1046/j.1538-7836.2003.00261.x

16. Zhang NN, Shi XP, Chen XW, et al. Association between inflammatory markers, hemostatic markers,and microvascular complications in 182 Chinese patients with type 2 diabetes mellitus. Science. 2015;46 (3):214-220.

17. Zhang ZN, Hao YS, Zhao YQ, et al. Infectious diseases and hematological abnormalities. Hematology. 2013:1440-1445.

18. Yan M, Cheng X, Lu Y, et al. [Relationship between different levels of C-reactive protein and IL-6 and anemia in cancer patients]. Zhong Guo Ji Xu Yi Xие Jiao Yu. 2018;10(34):76-78. Chinese.

19. Cheng Z, Yan M, Lu Y, et al. Expression of serum BMP6 and hepcidin in cancer-related anemia. Hematology. 2020;25 (1):134-138. doi:10.1080/16078454.2020.1738098
International Journal of General Medicine

\section{Publish your work in this journal}

The International Journal of General Medicine is an international, peer-reviewed open-access journal that focuses on general and internal medicine, pathogenesis, epidemiology, diagnosis, monitoring and treatment protocols. The journal is characterized by the rapid reporting of reviews, original research and clinical studies across all disease areas. The manuscript management system is completely online and includes a very quick and fair peer-review system, which is all easy to use. Visit http://www.dovepress.com/ testimonials.php to read real quotes from published authors. 